\title{
Inhibitors of the PD-I/PD-LI axis for the treatment of head and neck cancer: current status and future perspectives
}

This article was published in the following Dove Press journal:

Drug Design, Development and Therapy

3 July 2017

Number of times this article has been viewed

\section{Xiongwen Ran \\ Kai Yang}

Department of Oral and Maxillofacial Surgery, The First Affiliated Hospital of Chongqing Medical University, Chongqing, People's Republic of China

Correspondence: Kai Yang Department of Oral and Maxillofacial Surgery, The First Affiliated Hospital of Chongqing Medical University, I Youyi Road, Yuzhong District, Chongqing 4000 16, People's Republic of China Tel +8623890l 2569

Email cqfyyk@hotmail.com
Abstract: Head and neck cancer (HNC) is a common malignant tumor, but traditional therapeutic methods have unsatisfactory curative effects and many complications occur. Hence, there is an urgent need to develop therapeutic methods that can elicit curative effects as well as low toxic and few side effects. With the development of cancer molecular biology and immunology, targeted therapy for immune checkpoints of programmed cell death 1 (PD-1) and programmed cell death ligand 1 (PD-L1) has shown enormous development prospects for HNC treatment. Groundbreaking progress has been achieved in the treatment of recurrent/metastatic head and neck squamous cell carcinoma (HNSCC). This review describes current treatment by PD-1- and PD-L1-targeted drugs for HNC.

Keywords: head and neck cancer, squamous cell carcinoma, programmed cell death 1, programmed cell death ligand 1, immunotherapy, immune checkpoint

\section{Introduction}

Head and neck cancer (HNC) is the eighth most common cancer worldwide. Approximately $90 \%$ of $\mathrm{HNC}$ is head and neck squamous cell carcinoma (HNSCC), with $\approx 650,000$ new cases reported annually worldwide. ${ }^{1,2}$ As a rule, HNC is divided into two subclasses: human papillomavirus positive (HPV+) and human papillomavirus negative (HPV-). ${ }^{3} \mathrm{HPV}-\mathrm{HNC}$ is caused mostly by tobacco smoking and alcoholism ( $\approx 75 \%$ of patients), and these patients have stronger resistance to treatment and a worse prognosis than HPV+ HNC patients. $^{2-8}$

Despite continuous development of conventional therapies such as surgery, radiotherapy, and chemotherapy, the 5-year survival of HNSCC is worse than that of other cancers. Since 1975, the 5-year relative survival for cancer of the oral cavity and pharynx has increased from $53 \%$ to $63 \%$, whereas that of laryngeal cancer has decreased from $67 \%$ to $61 \% .^{9-12}$ In addition, conventional therapeutic methods can result in serious physiologic and psychological complications such as pain, infection, caries, distress, depression, or anxiety.,13 Therefore, the development of new therapeutic methods of high curative value as well as low toxicity and side effects, especially for patients with advanced HNC, is needed.

Thanks to in-depth studies on the molecular biology and immunology of cancer, cancer immunotherapy has shown immense prospects for the development in HNSCC treatment. The effector arm of cancer immunotherapy is regulated by positive and negative co-signaling pathways or "immunologic checkpoints." The checkpoints of programmed cell death 1 (PD-1) and programmed cell death ligand 1 (PD-L1) have 
important roles in the formation of "immune privilege" regions, viral persistence, tumor development, and immune evasion. ${ }^{2,14,15}$ In 2016, the results of two milestone trials an open-label, multicenter, Phase Ib trial (KEYNOTE-012) and a randomized, open-label, Phase III trial (CheckMate $141)^{16-18}$ - were announced. Those results facilitated pembrolizumab (MK-3475) and nivolumab (BMS-936558), which are antagonists of the PD-1/PD-L1 axis, to become the first molecular-targeted therapeutic drugs to be approved by the US Food and Drug Administration (FDA) for HNSCC. Later, the two drugs were compiled into clinical guidelines for HNSCC treatment in the National Comprehensive Cancer Network and became the fourth standard therapeutic method (after surgery, radiotherapy, and chemotherapy) for HNSCC. ${ }^{19}$ These observations suggest that moleculartargeted therapy for HNSCC may be entering a new era.

The current status of anti-PD-1/anti-PD-L1-targeted drugs in HNC treatment is summarized in this review. Also, a prospective evaluation of PD-1/PD-L1 molecular-targeted therapy is presented.

\section{Structural features of PD-I/PD-LI}

PD-1 is a member of the CD28/CTLA-4/ICOS costimulatory receptor family. PD-1 is a monomeric type-I surface transmembrane glycoprotein consisting of 268 amino acids. Its structure mainly comprises an extracellular region consisting of a higher signal sequence and immunoglobulin variable (IgV)-like structures, a hydrophobic transmembrane region, and an intracellular region. There are two independent tyrosine residues at the tail end in the intracellular region. The tyrosine residue at the amino end participates to constitute an immunoreceptor tyrosine-based inhibitory motif. The tyrosine residue at the hydroxyl end helps to constitute an immunoreceptor tyrosine-based switch motif (ITSM). ${ }^{20-22}$

The PD-1 ligands termed PD-L1 and PD-L2 belong to the B7 superfamily, and the family members also include B7-1 (cluster of differentiation [CD]80), B7-2 (CD86), B7-H2, B7-H3, B7-H4, and B7-H6. ${ }^{1423}$ The structures of PD-L1 and PD-L2 mainly comprise an extracellular region consisting of IgV-like structures in series and immunoglobulin constant region-like structures, a hydrophobic transmembrane region, and an intracellular region with a short cytoplasmic tail of unknown function. ${ }^{20,23}$ The affinity of the PD-L1 extracellular region with PD-1 is lower than that of the PD-L2 extracellular region with $\mathrm{PD}-1$. However, the PD-L1 extracellular region can also bind with the $\mathrm{B} 7-\mathrm{A}(\mathrm{CD} 80)$ extracellular region. ${ }^{14,20,23}$ PD-L2 is expressed only in activated macrophages and dendrites, ${ }^{20}$ hence, only few studies have been done on PD-L2. Therefore, the emphasis of this review is on PD-1 and PD-L1.

\section{Expression pattern and antitumor mechanism of PD-I/PD-LI and their expression profile in HNSCC Expression pattern of PD- I/PD-LI}

PD-1 is expressed on activated T cells after induction by a T-cell antigen receptor and cytokine receptor. ${ }^{20} \mathrm{PD}-1$ is also expressed at low levels on double-negative (CD4-CD8 $\left.{ }^{-}\right)$ $\mathrm{T}$ cells in the thymus, activated natural killer T cells, B cells, monocytes, and immature Langerhans' cells. ${ }^{14,20,22}$ Transcription of PD-1 in T cells requires nuclear translocation of nuclear factor of activated T cells (NFAT) as well as binding of NFATc1 (NFAT2) to the PDCD1 promoter. ${ }^{20}$

PD-L1 is expressed constitutively at low levels on antigen-presenting cells (APCs) and a wide variety of nonhematopoietic cell types, including vascular endothelial cells, pancreatic islet cells, and cells at sites of immune privilege (eg, placenta, testes, and eye). ${ }^{14,20,24}$ Inflammatory cytokines such as type I and type II interferons as well as tumor necrosis factor $\alpha$ and vascular endothelial growth factor can induce PD-L1 expression..$^{20,24}$

Tumor cells upregulate PD-L1 expression by four main mechanisms. The first mechanism is activation of the epidermal growth factor receptor (EGFR), mitogen-activated protein kinase, phosphoinositide 3-kinase (PI3K)-Akt or Janus kinase 2/signal transducer and activator of transcription 1 signaling pathways. ${ }^{20,26-28}$ Increased expression of STAT3 and hypoxia-inducible factor- 1 transcription factors can upregulate the expression of PD-L1. ${ }^{25,29-31}$ The second mechanism is amplification of genes coding PD-L1 (9p24.1). ${ }^{20,26}$ The third mechanism is induction of the Epstein-Barr virus (EBV). Gastric cancer and EBV + nasopharyngeal carcinoma can result in high expression of PD-L1 even without amplification of the $9 p 24.1$ gene. ${ }^{32,33}$ However, whether the HPV is positive in HNSCC is not correlated with PD-L1 expression; ${ }^{27,34}$ the final mechanism is epigenesis. Some microRNAs (miR-34a, miR-200, miR-513, and miR-570) have a negative correlation with PD-L1 expression. ${ }^{20,35}$ Moreover, in the tumor microenvironment, the stimulatory effects of inflammatory factors can also induce PD-L1 expression, where interferon- $\gamma$ is the most important stimulating factor. ${ }^{15,20,24,25,27}$

\section{Antitumor mechanism of PD-I/PD-LI}

Complete activation of $\mathrm{T}$ cells is dependent upon the regulation of a "dual-signal" system. The first signal is derived from specific binding between a $\mathrm{T}$-cell receptor and a major 
histocompatibility complex class, namely, antigen recognition of T cells. The second signal arises from co-stimulating molecules, namely, the signal mediated by the interaction between APC-expressed co-stimulatory molecules and the corresponding receptor or ligand on the T-cell surface. For example, CD28/B7 is an important positive co-stimulating molecule. ${ }^{14,20,21,36}$ In addition to ensuring that $\mathrm{T}$ cells are not overstimulated, there are negative co-stimulatory molecules that regulate $\mathrm{T}$ cells, and they are mainly cytotoxic T-lymphocyte-associated protein 4 (CTLA4)-B7 signaling pathways and PD-1/PD-L1 signaling pathways. ${ }^{14,21,22,24}$ After PD-1 and PD-L1 bind with each other in activated T cells, tyrosine in the ITSM structural domain of PD-1 undergoes phosphorylation, which causes dephosphorylation of the downstream protein kinases Syk and PI3K. These actions lead to inhibition of the activation of downstream channels such as Akt and ERK. Finally, inhibition of the transcription and translation of genes and cytokines required by $\mathrm{T}$-cell activation leads to the regulation of T-cell activity. ${ }^{20}$ After invasion by tumor cells, these signal channels are used to inhibit T-cell activation so as to evade attack by the immune system. At present, inhibitors of immune checkpoints have been studied, and the ones applied most extensively are CTLA-4, PD-1, and PD-L1 monoclonal antibodies. The antitumor effect is realized by the inhibition of the activity of immune checkpoints, blockade of immunosuppression in the tumor microenvironment, and reactivation of the immune response of T cells to the tumor (Figure 1). ${ }^{14-18,20,22,24,37}$

\section{Expression profile of PD-I/PD-LI in HNSCC}

Increased expression of PD-1/PD-L1 in the microenvironment of HNSCC is independent of HPV status. Yu et a ${ }^{34}$ undertook a meta-analysis of 18 data sets of gene expression

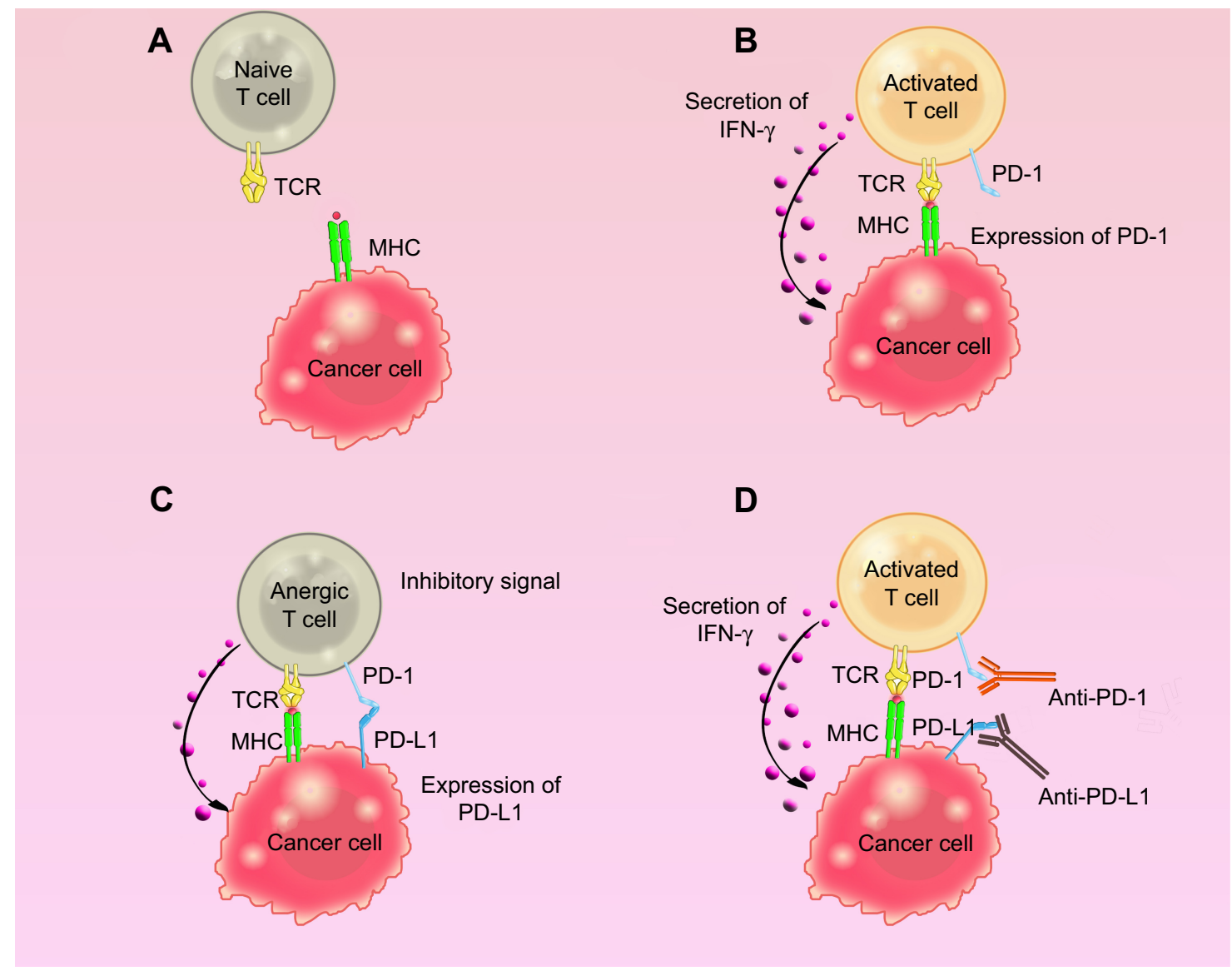

Figure I Mechanism of adaptive immune resistance in the blockade of PD-I/PD-LI pathway.

Notes: (A) Naive T cells around cancer cells were collected. (B) The TCR recognized and activated T cells with MHC, and besides, they induced T cells to express PD-I and secrete IFN. (C) Local levels of IFN increased to induce PD-I expression in cancer cells. PD-LI and T-cell-expressed PD-I recognized and generated an inhibitory signal, and as a result, the activated T cells lost their activity. (D) Application of PD-I/PD-LI antibody drugs blocked the PD-I/PD-LI signaling pathway and removed the inhibitory signal, allowing the $T$ cells to attack the tumor cell.

Abbreviations: TCR, T-cell receptor; MHC, major histocompatibility complex; IFN- $\gamma$, interferon $\gamma$; PD-I, programmed cell death I; PD-LI, programmed cell death ligand I; Anti-PD-I, antibody programmed cell death I; anti-PD-LI, antibody programmed cell death ligand I. 
of HNCs and verified that CD274 (the gene encoding PD-L1) and $C D 279$ (the gene encoding PD-1) DNA copy numbers, as well as the mRNA expression of these genes, was increased significantly in HNSCC $(P<0.05)$. Also, through a comparative analysis of HNSCC and the normal mucosa, PD-1/ PD-L1 expression in cells was high in the microenvironment of HNSCC, but there was no obvious difference in HPV+ $(n=12)$ or HPV- $(n=74)$ subgroups.

In a tumor microenvironment, PD-L1 is expressed not only on relevant immune cells in the microenvironment but also on tumor cytomembranes and in the cytoplasm. LyfordPike et $\mathrm{al}^{24}$ compared HPV-infected non-cancerous adult tonsil tissues with cancer tissues from HNSCC patients and verified that, at the cell level, localized expression of PD-L1 was within deep tonsillar crypts, the site of initial HPV infection, and the origin of HPV-HNSCC. PD-L1 in tumor cells was expressed mainly on membranes (cell surface) and partially in the cytoplasm.

\section{Clinical application of PD- I/PD-LI - targeted drugs for HNC treatment}

Currently, the PD-1/PD-L1-targeted drugs used in HNC treatment are pembrolizumab, nivolumab, and durvalumab (Table 1).

\section{PD-I-targeted drugs}

The main PD-1-targeted drugs used to treat HNSCC are pembrolizumab and nivolumab, both of which are humanized
PD-1-inhibiting IgG4 monoclonal antibodies with high specificity. ${ }^{16-18}$ They have been used for the treatment of patients with recurrent/metastatic HNSCC with disease progression on or after platinum-containing chemotherapy. Also, pembrolizumab has certain effects on adult patients with salivary gland carcinomas at an advanced stage. ${ }^{38}$

\section{Pembrolizumab}

Seiwert et $a l^{16}$ used pembrolizumab to treat 60 patients with recurrent/metastatic HNSCC. All the patients had PD-L1 expression in tumor cells of $\geq 1 \%$, whereas 23 cases $(38 \%)$ were HPV+ and 37 cases (62\%) were HPV-. Pembrolizumab (10 mg/kg body weight, intravenous [iv]) was administered once every 2 weeks for 24 months. Median duration of follow-up was 14 (interquartile range [IQR] 4-14) months. The overall response rate (ORR) was $18 \%$, whereas the response rate of $\mathrm{HPV}+$ was $25 \%$ and that of HPV- was $14 \%$. The median progression-free survival (PFS) was 2 months. The median overall survival (OS) was 13 months. Thirtyeight cases experienced treatment-related adverse events (63\%), mainly fatigue, pruritus, nausea, reduced appetite, and rash. There were no treatment-related deaths.

Chow et $\mathrm{al}^{17}$ used pembrolizumab to treat 132 adult patients with recurrent/metastatic HNSCC. Of these, 28 cases (21\%) were HPV+ and 104 cases (71\%) were HPV-. Pembrolizumab (200 mg, iv) was administered once every 3 weeks for 24 months. The median duration of follow-up was 9 (IQR 3-11) months. The ORR was 18\%, and the response

Table I Efficacy and safety of PD-I/PD-LI-targeted drugs in HNC treatment

\begin{tabular}{|c|c|c|c|c|c|c|c|c|}
\hline Monoclonal antibody & Phase & $\begin{array}{l}\text { Patient } \\
\text { no }\end{array}$ & ORR, no (\%) & $\begin{array}{l}\text { PFS } \\
\text { (months) }\end{array}$ & $\begin{array}{l}\text { OS } \\
\text { (months) }\end{array}$ & $A E$, no (\%) & $A E \geq 3$, no (\%) & DrD no \\
\hline $\begin{array}{l}\text { Pembrolizumab } \\
(\text { MK-3475) }\end{array}$ & $\mathrm{lb}$ & 60 & $8(17.8 \%)$ & 2 & 13 & $38(63 \%)$ & $10(17 \%)$ & 0 \\
\hline $\begin{array}{l}\text { Pembrolizumab } \\
(\text { MK-3475) }\end{array}$ & $\mathrm{lb}$ & 132 & $24(18.2 \%)$ & 3 & 8 & $82(62 \%)$ & $12(9 \%)$ & 0 \\
\hline $\begin{array}{l}\text { Pembrolizumab } \\
(\text { MK-3475) }\end{array}$ & II & 50 & $9(18.0 \%)$ & - & - & 35 (70\%) & $6(12 \%)$ & I \\
\hline $\begin{array}{l}\text { Pembrolizumab } \\
(\text { MK-3475) }\end{array}$ & $\mathrm{lb}$ & 26 & $3(11.5 \%)$ & - & - & $22(84.6 \%)$ & $3(11.5 \%)$ & 1 \\
\hline $\begin{array}{l}\text { Durvalumab } \\
\text { (MEDI4736) }\end{array}$ & $1 / I I$ & 62 & $6(11.7 \%)$ & - & - & $60 \%$ & $7 \%$ & 0 \\
\hline $\begin{array}{l}\text { Durvalumab } \\
\text { (MEDI4736) }\end{array}$ & I & 50 & - & - & - & $39 \%$ & $5 \%$ & 0 \\
\hline $\begin{array}{l}\text { Durvalumab } \\
\text { (MEDI4736) }\end{array}$ & I & 105 & - & - & - & $33 \%$ & $7 \%$ & - \\
\hline $\begin{array}{l}\text { Nivolumab } \\
\text { (BMS-936558) }\end{array}$ & III & 240 & $32(13.3 \%)$ & 2.0 & 7.5 & 139 (58.9\%) & 31 (13.1\%) & 2 \\
\hline Standard therapy & III & 121 & $7(5.8 \%)$ & 2.3 & 5.1 & $86(77.5 \%)$ & $39(35.1 \%)$ & 1 \\
\hline
\end{tabular}

Notes: '-' indicates no mention in the original literature. ${ }^{~ P e m b r o l i z u m a b ~(10 ~ m g / k g, ~ i n t r a v e n o u s) ~ o n c e ~ e v e r y ~} 2$ weeks. ${ }^{\mathrm{b}}$ Pembrolizumab (200 mg, intravenous) once every 3 weeks.

Abbreviations: HNC, head and neck cancer; ORR, overall response rate; PFS, progression-free survival; OS overall survival; AE, adverse events; DrD, drug-related death. 
rate of HPV+ was $32 \%$ and that of HPV- was $14 \%$. The median PFS was 2 months. The median OS was 8 months. Also, 82 cases (62\%) experienced treatment-related adverse events, mainly fatigue, hypothyroidism, and reduced appetite. There were no treatment-related deaths.

Bauml et $\mathrm{al}^{39}$ used pembrolizumab to treat 50 patients with recurrent/metastatic HNSCC after the failure of platinumbased treatment and cetuximab. Pembrolizumab (200 mg, iv) was administered once every 3 weeks for 24 months. The median duration of follow-up was 6.8 months. The ORR was $18 \%$; the partial response rate (PRR) was $18 \%$ and the stable disease rate was $18 \%$. Also, 35 cases $(70 \%)$ experienced treatment-related adverse events, mainly specific immunologic diseases, hypothyroidism, and pneumonia. Two patients (4\%) discontinued treatment because of treatment-related adverse events, and 1 patient ( $2 \%$ ) died of treatment-related adverse events. Their clinical trial showed that pembrolizumab also had high antitumor activity and few toxic and side effects for patients with recurrent/metastatic HNSCC who showed tolerance to previous treatment.

Cohen ${ }^{38}$ used pembrolizumab to treat 26 adult patients with advanced carcinoma of the salivary glands. Pembrolizumab (10 mg/kg, iv) was administered once every 2 weeks for 24 months. The median duration of follow-up was 61.9 (IQR, 8.7-88.4) weeks. The ORR was 11.5\%; $20.7 \%$ of patients reached 6-month PFS. A total of $70.4 \%$ patients reached 6-month OS. Also, 22 patients (84.6\%) experienced treatment-related adverse events, mainly diarrhea, reduced appetite, pruritus, and fatigue. Two patients discontinued treatment because of arthritis and hepatitis, and 1 patient died of interstitial pneumonia. Their clinical trial showed that pembrolizumab had high antitumor activity and few toxic and side effects for HNC patients with carcinoma of the salivary glands at middle and advanced stages.

\section{Nivolumab}

Ferris et $\mathrm{al}^{18}$ randomly divided 361 adult patients with recurrent/metastatic HNSCC into a nivolumab group (240 cases) and standard therapy group (121 cases). The former was given nivolumab ( $3 \mathrm{mg} / \mathrm{kg}$, iv) once every 2 weeks, whereas the latter was given a traditional agent (methotrexate, docetaxel, or cetuximab), and the clinical trial continued until disease progression. Responses were assessed every 6 weeks and the median duration of follow-up was 5.1 months. The ORR and PRR of the nivolumab group were $13.3 \%$ and $10.8 \%$, and those of the standard therapy group were $5.8 \%$ and $5.0 \%$, respectively. The median PFS of the nivolumab group was 2.0 months, and $19.7 \%$ patients reached 6-month
PFS. The median PFS of the standard therapy group was 2.3 months and $9.9 \%$ patients reached 6-month PFS. The median OS of the nivolumab group was 7.5 months and $36 \%$ patients reached 1-year PFS. The median OS of the standard therapy group was 5.1 months and $16.6 \%$ patients reached 1-year PFS. Also, 139 patients in the nivolumab group experienced treatment-related adverse events (58.9\%), mainly fatigue, nausea, and rash. In addition, 31 patients (13.1\%) experienced treatment-related adverse events of grade $>3$, and 2 patients died of hypercalcemia and pneumonia, respectively. Eighty-six patients in the standard therapy group experienced treatment-related adverse events (77.5\%), mainly nausea, fatigue, and anemia. Also, 39 patients (35.1\%) experienced treatment-related adverse events of grade $>3$, and 1 patient died of pulmonary infection. The curative effect and survival period for nivolumab were more than twofold better and longer than a traditional regimen, and the number of serious adverse reactions was about one-third that of the traditional regimen.

\section{PD-LI-targeted drugs}

At present, the main PD-L1-targeted drug for HNSCC treatment is durvalumab (MEDI4736). It is a human IgG1 monoclonal antibody that blocks PD-L1 binding to its receptors ${ }^{14,40,41}$ and is used for recurrent/metastatic HNSCC.

Segal et $\mathrm{al}^{40}$ used durvalumab to treat 62 adult patients with recurrent/metastatic HNSCC who underwent PD-L1 immunohistochemical analyses. Overall, 40\% of patients were HPV+, $39 \%$ were HPV-, and $21 \%$ of patients could not be evaluated. Durvalumab (10 mg/kg, iv) was administered once every 2 weeks for 12 months. The median duration of follow-up was 24 weeks. Among 71 evaluable patients, the ORR was $12 \%$, whereas the response rate of PD-L1-positive patients was $25 \%$. The 24 -week disease control rate was $16 \%$ and that of PD-L1 positive patients was $25 \%$. The prevalence of treatment-related adverse events among these patients was $60 \%$ and was mainly fatigue, diarrhea, and nausea. Also, $7 \%$ of patients suffered treatment-related adverse events of grade $>3$. There were no treatment-related deaths.

Fury et $\mathrm{a}^{41}$ used durvalumab to treat 50 adult patients diagnosed with recurrent/metastatic HNSC. They were administered durvalumab ( $10 \mathrm{mg} / \mathrm{kg}$, iv) once every 2 weeks for 12 months. The median duration of follow-up was 8 weeks. Among the 29 patients assessed, 7 cases (24.1\%) had obvious radiographic shrinkage of target tumor lesions, whereas 5 did not have objective evidence of progression for $\geq 12$ (IQR, 6-24) weeks. Four patients showed a partial response. For evaluable patients with a median administration dose 
of 3 (IQR, 1-8) times, the prevalence of treatment-related adverse events was 39\%, mainly nausea, diarrhea, dizziness, and rash. Treatment-related adverse events of grade $>3$ was observed in $5 \%$ of patients and was associated with increased glutamyltransferase levels. No treatment-related adverse events led to study discontinuation.

Segal $^{42}$ used durvalumab to treat 151 adult patients with recurrent/metastatic HNSCC. They were given durvalumab (10 mg/kg, iv) once every 2 weeks for 12 months. Among 105 evaluable patients with a median administration dose of 3 (IQR, 1-8) times, the prevalence of treatment-related adverse events was $33 \%$. Also, $7 \%$ of patients experienced treatment-related adverse events of grade $>3$, mainly fatigue, nausea, and rash.

\section{Conclusion}

The clinical trials mentioned above show that PD-1/PDL1-targeted drugs have obvious advantages over traditional therapeutic regimens in terms of ORR, survival, and safety when treating recurrent/metastatic HNC. They have shown enormous potential as new types of anticancer drugs. The ORR of PD-1 as treatment of recurrent/metastatic HNC was higher than that of PD-L1. There was no significant difference between PD-1-targeted drugs and PD-L1-targeted drugs in terms of the prevalence of treatment-related adverse events. However, the prevalence of treatment-related adverse events of grade $>3$ by PD-1-targeted drugs was obviously higher than that observed with PD-L1-targeted drugs. We hypothesize that the reason for the differences between PD-1- and PD-L1-targeted drugs in curative effect and safety is that PD1 antibody can simultaneously block the signaling pathways between PD-1 and PD-L1 and between PD-1 and PD-L2. If the signaling pathway between PD-1 and PD-L1 is blocked, $\mathrm{T}$-cell inhibition will occur and its antitumor effect will be improved. Conversely, because PD-L2 mediates immune tolerance to protect the organism, blocked signaling pathways between PD-1 and PD-L1 may cause more serious adverse events. ${ }^{43,44}$ However, PD-L1 antibody can only block the signaling pathway between PD-1 and PD-L1 and not the one between PD-L2 and PD-1. Hence, the prevalence of severe adverse events caused by PD-L1 antibody is lower than that caused by PD-1 antibody. Based on the pre-condition that the prevalence of severe adverse responses is controlled effectively, PD-1-targeted drugs have greater potential than PD-L1-trargeted drugs. Among PD-1-targeted drugs, pembrolizumab has the highest ORR in HNSCC treatment and has the greatest potential among PD-1/PD-L1-targeting drugs. Pembrolizumab treatment leads to a better prognosis for HPV+ HNSCC patients than for HPV- HNSCC patients.
Moreover, pembrolizumab has higher clinical activity and a lower prevalence of treatment-related adverse events for HNSCC than for salivary gland carcinoma. Therefore, pembrolizumab has the greatest potential for the treatment of HPV+ HNSCC (Table 1).

The clinical trials stated above show that, when the regimens of fixed-dose pembrolizumab (200 mg, iv, once every 3 weeks) and a dose regimen based on weight ( $10 \mathrm{mg} / \mathrm{kg}$ once every 2 weeks) were compared, no significant differences were found in their ORR, PFS, and overall occurrence rate of treatment-related adverse events. Although a fixed-dose regimen carries a lower OS than a dose regimen by weight, it can greatly reduce the prevalence of adverse events of grade $>3$ and is more acceptable to patients. This may be the reason why a fixed-dose regimen $(200 \mathrm{mg}$, iv, for 30 minutes once every 3 weeks) is recommended by the FDA.

\section{Research prospects and expectations of PD-I/PD-LI-targeted treatment of $\mathrm{HNC}$}

Exclusive application of PD-1- or PD-L1-targeted drugs has achieved groundbreaking curative effects in the treatment of recurrent/metastatic HNSCC in clinical trials and has shown enormous development prospects. Even though exclusive application of PD-1- or PD-L1-targeted drugs for the treatment of recurrent/metastatic HNSCC has been approved by the FDA, several problems remain.

The curative effect and safety of PD-1/PD-L1-targeted therapy for recurrent/metastatic HNSCC requires more clinical data. So far, data have been obtained using a single drug, such as pembrolizumab, nivolumab, or durvalumab, to treat ungrouped recurrent/metastatic HNSCC. Experiments for grouped recurrent/metastatic HNSCC using two or three drugs in combination have not been done. Pembrolizumab has the greatest potential as a PD-1-targeted drug, but its optimal dose, administration regimen, and prevalence of treatment-related adverse events of grade $>3$ must be discovered.

Besides recurrent/metastatic HNSCC, the curative effect and safety of PD-1/PD-L1-targeted drugs for HNSCC and $\mathrm{HNC}$ of other diseases in different clinical stages should be explored further. PD-1/PD-L1-targeted drugs can be immunosuppressive to the organism. ${ }^{20}$ Then, whether PD-1/ PD-L1-targeted drugs have a favorable inhibitory effect on precancerous lesions and early phase or metaphase HNSCC and whether they can reduce local tumor recurrence or distant metastasis can be studied. Pembrolizumab has a favorable curative effect and safety for the treatment of advanced-stage salivary gland carcinoma, ${ }^{38}$ and EBV + nasopharyngeal 
carcinoma has high expression of PD-L1. ${ }^{32,33}$ These observations suggest that PD-1/PD-L1-targeted drugs may have good therapeutic effects in other HNC disorders, such as adenocarcinoma and nasopharyngeal carcinoma, which merits further research.

The curative effect and safety of PD-1/PD-L1-targeted drugs using a traditional regimen to treat HNSCC should be explored further. Several clinical trials have shown that PD-1/PD-L1-targeted drugs combined with radiotherapy and chemotherapy can enhance the killing of tumor cells. ${ }^{45-48}$ Hence, clinical research on PD-1/PD-L1-targeted drugs combined with traditional chemotherapeutics (docetaxel, cisplatin, and 5-fluorouracil), biotherapy (vorinostat, imlygic, and motolimod), radiotherapy (intensity-modulated radiotherapy, image-guided radiotherapy, and stereotactic body radiotherapy), and surgical treatment should be strengthened. Selection of drug doses, safety, and tolerability in combination therapy should be examined.

Also, an in-depth exploration of the curative effect and safety of PD-1/PD-L1-targeted drugs for HNSCC treatment should be carried out. PD-1/PD-L1-targeted drugs show their respective antitumor effects, but whether combined application of PD-1- and PD-L1-targeted drugs can elicit enhanced effects needs further experimental verification. Cetuximab is a monoclonal antibody of the targeted EGFR, and studies have shown that blockade of PD-1/PD-L1 signaling can improve anticancer effects. ${ }^{49}$ Hence, combined application of PD-1/PD-L1-targeted drugs and cetuximab may also have favorable clinical effects. Ipilimumab and tremelimumab are human-specific monoclonal antibodies that inhibit CTLA-4 expression, and studies have shown that simultaneous targeting of PD-L1 and CTLA-4 pathways generates an enhanced effect. ${ }^{50}$ Hence, the effect of a combined therapeutic schedule of PD-L1-target drugs and CTLA-4-targeted drugs merits further research.

\section{Acknowledgment}

We thank all the HNC patients who participated in these and other HNSCC clinical trials to improve patient care for the next generation.

\section{Disclosure}

The authors report no conflicts of interest in this work.

\section{References}

1. Ferlay J, Soerjomataram I, Dikshit R, et al. Cancer incidence and mortality worldwide: sources, methods and major patterns in GLOBOCAN 2012. Int J Cancer. 2015;136(5):E359-E386.

2. Dorsey K, Agulnik M. Promising new molecular targeted therapies in head and neck cancer. Drugs. 2013;73(4):315-325.
3. Westra WH. The changing face of head and neck cancer in the 21 st century: the impact of HPV on the epidemiology and pathology of oral cancer. Head Neck Pathol. 2009;3(1):78-81.

4. Cohen EE, LaMonte SJ, Erb NL, et al. American Cancer Society Head and Neck Cancer Survivorship Care Guideline. CA Cancer J Clin. 2016;66(3):203-239.

5. Gillison ML, Chaturvedi AK, Anderson WF, Fakhry C. Epidemiology of human papillomavirus-positive head and neck squamous cell carcinoma. J Clin Oncol. 2015;33(29):3235-3242.

6. Simard EP, Ward EM, Siegel R, Jemal A. Cancers with increasing incidence trends in the United States: 1999 through 2008. CA Cancer J Clin. 2012;62(2):118-128.

7. Dillon MT, Harrington KJ. Human papillomavirus-negative pharyngeal cancer. J Clin Oncol. 2015;33(29):3251-3261.

8. Benson E, Li R, Eisele D, Fakhry C. The clinical impact of HPV tumor status upon head and neck squamous cell carcinomas. Oral Oncol. 2014;50(6):565-574.

9. National Comprehensive Cancer Network [webpage on the Internet] NCCN Clinical Practice Guidelines in Oncology (NCCN Guidelines ${ }^{\circledR}$ ) Head and Neck [serial on the Internet]. 2015; Version 1. Available from: https://www.nccn.org/professionals/physician_gls/f_guidelines. asp\#head-and-neck. Accessed June 6, 2016.

10. Grégoire V, Lefebvre JL, Licitra L, Felip E; EHNS-ESMO-ESTRO Guidelines Working Group. Squamous cell carcinoma of the head and neck: EHNS-ESMO-ESTRO Clinical Practice Guidelines for diagnosis, treatment and follow-up. Ann Oncol. 2010;21(Suppl 5): v184-v186.

11. Jemal A, Siegel R, Ward E, Hao Y, Xu J, Thun MJ. Cancer statistics, 2009. CA Cancer J Clin. 2009;59(4):225-249.

12. Miller KD, Siegel RL, Lin CC, et al. Cancer treatment and survivorship statistics, 2016. CA Cancer J Clin. 2016;66(4):271-289.

13. Epstein JB, Thariat J, Bensadoun R-J, et al. Oral complications of cancer and cancer therapy: from cancer treatment to survivorship. CA Cancer J Clin. 2012;62(6):400-422.

14. Pai SI, Zandberg DP, Strome SE. The role of antagonists of the PD-1:PD-L1/PD-L2 axis in head and neck cancer treatment. Oral Oncol. 2016;61:152-158.

15. Pai SI. Adaptive immune resistance in HPV-associated head and neck squamous cell carcinoma. Oncoimmunology. 2013;2(5):e24065.

16. Seiwert TY, Burtness B, Mehra R, et al. Safety and clinical activity of pembrolizumab for treatment of recurrent or metastatic squamous cell carcinoma of the head and neck (KEYNOTE-012): an open-label, multicentre, phase 1b trial. Lancet Oncol. 2016;17(7):956-965.

17. Chow LQ, Haddad R, Gupta S, et al. Antitumor activity of pembrolizumab in biomarker-unselected patients with recurrent and/or metastatic head and neck squamous cell carcinoma: results from the phase Ib KEYNOTE-012 expansion cohort. J Clin Oncol. 2016;34(32): 3838-3845.

18. Ferris RL, Blumenschein G, Fayette J, et al. Nivolumab for recurrent squamous-cell carcinoma of the head and neck. N Engl J Med. 2016; 375(19):1856-1867.

19. National Comprehensive Cancer Network [webpage on the Internet]. NCCN Clinical Practice Guidelines in Oncology (NCCN Guidelines ${ }^{\circledR}$ ) Head and Neck [serial on the Internet]. 2016; Version 2. Available from: https://www.nccn.org/professionals/physician_gls/f_guidelines. asp\#head-and-neck. Accessed December 3, 2016.

20. Boussiotis VA. Molecular and biochemical aspects of the PD-1 checkpoint pathway. $N$ Engl J Med. 2016;375(18):1767-1778.

21. Zhang X, Schwartz JC, Guo X, et al. Structural and functional analysis of the costimulatory receptor programmed death-1. Immunity. 2004; 20(3):337-347.

22. Cheng X, Veverka V, Radhakrishnan A, et al. Structure and interactions of the human programmed cell death 1 receptor. J Biol Chem. 2013; 288(17):11771-11785.

23. Yao Q, Fischer KP, Tyrrell DL, Gutfreund KS. The Peking duck programmed death-ligand 1: cDNA cloning, genomic structure, molecular characterization and mRNA expression analysis. Int J Immunogenet. 2015;42(2):111-120. 
24. Lyford-Pike S, Peng S, Young GD, et al. Evidence for a role of the PD-1:PD-L1 pathway in immune resistance of HPV-associated head and neck squamous cell carcinoma. Cancer Res. 2013;73(6):1733-1741.

25. Straub M, Drecoll E, Pfarr N, et al. CD274/PD-L1 gene amplification and PD-L1 protein expression are common events in squamous cell carcinoma of the oral cavity. Oncotarget. 2016;7(11):12024-12034.

26. Green MR, Monti S, Rodig SJ, et al. Integrative analysis reveals selective 9p24.1 amplification, increased PD-1 ligand expression, and further induction via JAK2 in nodular sclerosing Hodgkin lymphoma and primary mediastinal large B-cell lymphoma. Blood. 2010;116(17):3268-3277.

27. Akbay EA, Koyama S, Carretero J, et al. Activation of the PD-1 pathway contributes to immune escape in EGFR-driven lung tumors. Cancer Discov. 2013;3(12):1355-1363.

28. Concha-Benavente F, Srivastava RM, Trivedi S, et al. Identification of the cell-intrinsic and -extrinsic pathways downstream of EGFR and IFN $\gamma$ that induce PD-L1 expression in head and neck cancer. Cancer Res. 2016;76(5):1031-1043.

29. Marzec M, Zhang Q, Goradia A, et al. Oncogenic kinase NPM/ALK induces through STAT3 expression of immunosuppressive protein CD274 (PD-L1, B7-H1). Proc Natl Acad Sci U S A. 2008;105(52): 20852-20857.

30. Lee Y, Shin JH, Longmire M, et al. CD44+ cells in head and neck squamous cell carcinoma suppress T cell-mediated immunity by selective constitutive and inducible expression of PD-L1. Clin Cancer Res. 2016;(14):3571-3581.

31. Noman MZ, Desantis G, Janji B, et al. PD-L1 is a novel direct target of HIF-1 $\alpha$, and its blockade under hypoxia enhanced MDSC-mediated T cell activation. J Exp Med. 2014;211(5):781-790.

32. Fang W, Zhang J, Hong S, et al. EBV-driven LMP1 and IFN- $\gamma$ upregulate PD-L1 in nasopharyngeal carcinoma: implications for oncotargeted therapy. Oncotarget. 2014;5(23):12189-12202.

33. Derks S, Liao X, Chiaravalli AM, et al. Abundant PD-L1 expression in Epstein-Barr virus-infected gastric cancers. Oncotarget. 2016;7(22): 32925-32932.

34. Yu GT, Bu LL, Huang CF, et al. PD-1 blockade attenuates immunosuppressive myeloid cells due to inhibition of CD47/SIRP $\alpha$ axis in HPV negative head and neck squamous cell carcinoma. Oncotarget. 2015;6(39):42067-42080.

35. Chen J, Jiang CC, Jin L, Zhang XD. Regulation of PD-L1: a novel role of pro-survival signalling in cancer. Ann Oncol. 2016;27(3):409-416.

36. Moreno BH, Parisi G, Robert L, Ribas A. Anti-PD-1 therapy in melanoma. Semin Oncol. 2015;42(3):466-473.

37. Ibrahim R, Stewart R, Shalabi A. PD-L1 blockade for cancer treatment: MEDI4736. Semin Oncol. 2015;42(3):474-483.
38. Cohen RB. Preliminary results for the advanced salivary gland carcinoma cohort of the phase $1 \mathrm{~b}$ KEYNOTE-028 study of pembrolizumab. J Clin Oncol. 2016; ASCO meeting abstracts: 34(Supp1): abstr 6017.

39. Bauml J. Preliminary results from KEYNOTE-055: Pembrolizumab after platinum and cetuximab failure in head and neck squamous cell carcinoma (HNSCC). J Clin Oncol. 2016: ASCO meeting abstracts: 34(Suppl): abstr 6011.

40. Segal NH. Safety and efficacy of MEDI4736, an anti-PD-L1 antibody, in patients from a squamous cell carcinoma of the head and neck (SCCHN) expansion cohort. J Clin Oncol. 2015: ASCO meeting abstracts: 33(Suppl): abstr 3011.

41. Fury M, Ou SI, Balmanoukian A, et al. Clinical activity and safety of medi4736, an anti-PD-L1 antibody, in patients with head and neck cancer. Ann Oncol. 2014:25(Suppl 4):iv341.

42. Segal NH. Preliminary data from a multi-arm expansion study of MEDI4736, an anti-PD-L1 antibody. J Clin Oncol. 2014: ASCO meeting abstracts: $32(5 \mathrm{~s}$, Suppl); abstr 3002.

43. Akbari O, Stock P, Singh AK, et al. PD-L1 and PD-L2 modulate airway inflammation and iNKT-cell-dependent airway hyperreactivity in opposing directions. Mucosal Immunol. 2010;3(1):81-91.

44. Matsumoto K, Inoue $\mathrm{H}$, Nakano T, et al. B7-DC regulates asthmatic response by an IFN-gamma-dependent mechanism. J Immunol. 2004; 172(4):2530-2541.

45. Liniker E, Menzies AM, Kong BY, et al. Activity and safety of radiotherapy with anti-PD-1 drug therapy in patients with metastatic melanoma. Oncoimmunology. 2016;5(9):e1214788.

46. Kanda S, Goto K, Shiraishi H, et al. Safety and efficacy of nivolumab and standard chemotherapy drug combination in patients with advanced non-small-cell lung cancer: a four arms phase Ib study. Ann Oncol. 2016;27(12):2242-2250.

47. Ahmed KA, Stallworth DG, Kim Y, et al. Clinical outcomes of melanoma brain metastases treated with stereotactic radiation and anti-PD-1 therapy. Ann Oncol. 2016;27(3):434-441.

48. Deng L, Liang H, Burnette B, et al. Irradiation and anti-PD-L1 treatment synergistically promote antitumor immunity in mice. J Clin Invest. 2014;124(2):687-695.

49. Jie HB, Srivastava RM, Argiris A, et al. Increased PD-1+ and TIM-3+ TILs during cetuximab therapy inversely correlate with response in head and neck cancer patients. Cancer Immunol Res. 2017;5(5): 408-416.

50. Antonia S, Goldberg SB, Balmanoukian A, et al. Safety and antitumour activity of durvalumab plus tremelimumab in non-small cell lung cancer: a multicentre, phase 1b study. Lancet Oncol. 2016;17(3): 299-308.
Drug Design, Development and Therapy

\section{Publish your work in this journal}

Drug Design, Development and Therapy is an international, peerreviewed open-access journal that spans the spectrum of drug design and development through to clinical applications. Clinical outcomes, patient safety, and programs for the development and effective, safe, and sustained use of medicines are the features of the journal, which

\section{Dovepress}

has also been accepted for indexing on PubMed Central. The manuscript management system is completely online and includes a very quick and fair peer-review system, which is all easy to use. Visit http://www.dovepress.com/testimonials.php to read real quotes from published authors. 